\title{
Cellular cytokine and chemokine responses to parasite antigens and fungus and mite allergens in children co-infected with helminthes and protozoa parasites
}

Jana Hegewald ${ }^{1,2}$, Richard G Gantin², Christian J Lechner ${ }^{1,2}$, Xiangsheng Huang ${ }^{1,2}$, Abram Agosssou $^{3}$, Yvon F Agbeko $^{3}$, Peter T Soboslay ${ }^{1,2}$ and Carsten Köhler ${ }^{1,2^{*}}$

\begin{abstract}
Background: In sub-Saharan Africa poly-parasite infections are frequently observed in children, and with poly-parasitism modulating immune mechanisms, mediated by cytokines and chemokines, are required to prevent overwhelming inflammation and host tissue damage. We analyzed in children co-infected with helminthes and protozoan parasites their cellular production of regulatory and pro-inflammatory cytokines and chemokines in response to parasite antigens and allergens.
\end{abstract}

Methods: Intestinal and intravascular parasite infections were detected in stool and urines samples. The in vitro cellular cytokine and chemokine responses of peripheral blood mononuclear cells (PBMC) to parasite antigens and allergens were analysed in children $(n=87)$ with single and poly-parasite infection, and skin prick test reactivity to fungus and mite allergens was determined in singly and poly-parasitized children $(n=509)$.

Results: In children Entamoeba histolytica/dispar (62\%), Necator americanus (31\%), Schistosoma haematobium (28\%), S. mansoni (21\%), Hymenolepis nana (2\%) and Strongyloides stercoralis (1\%) were diagnosed. Singly infected were 37\%, $47 \%$ were positive for 2 or more parasite species and 16\% were infection-free. When PBMC were stimulated in vitro with parasite antigens and allergens, regulatory-type cytokine IL-27 and alarmin-type IL-33 enhanced with poly-parasite infections whilst IL-10 and pro-inflammatory MIP3-a/CCL20 and MIG/CXCL9 were produced in similar amounts in singly or poly-parasitized children. The co-stimulation in vitro of PBMC with mite allergens and Ascaris lumbricoides antigens depressed the allergen-induced pro-inflammatory IL-27, IL-33 and MIP3-a/CCL20 responses while regulatory IL-10 remained unaffected. Post albendazole and/or praziquantel treatment, the cellular release of IL-10, IL-33, MIP3-a/CCL20 and MIG/CXCL9 lessened significantly in all children infection groups. Skin prick test (SPT) reactivity to fungus Aspergillus fumigatus and mite Dermatophagoides pteronyssinus allergens was investigated in 509 children, and positive SPT responses were found in $23 \%$ of the infection-free, and in $47 \%, 53 \%$ and $56 \%$ of the singly, doubly and poly-parasite infected, respectively.

Conclusions: In children co-infected with helminthes and protozoan parasites a mixed cellular response profile of both inflammatory and regulatory chemokines and cytokines was stimulated by individual antigens and allergens, pro-inflammatory cytokines and chemokines enhanced with an increasing number of parasite infections, and in poly-parasitized children skin prick test reactivity to allergens extracts was highest.

Keywords: Schistosomiasis, Amoebiasis, Hookworm, Co-infection, Cytokine, Chemokine, Allergen, Skin prick test

\footnotetext{
* Correspondence: carsten.koehler@uni-tuebingen.de

${ }^{1}$ Institute for Tropical Medicine, University Clinics Tübingen, Wilhelmstraße

27, 72074 Tübingen, Germany

${ }^{2}$ Institut National d'Hygiène - Onchocerciasis Reference Laboratory, Sokodé,

Togo

Full list of author information is available at the end of the article
}

\section{Biomed Central}

(c) 2015 Hegewald et al.; licensee BioMed Central. This is an Open Access article distributed under the terms of the Creative Commons Attribution License (http://creativecommons.org/licenses/by/2.0), which permits unrestricted use, distribution, and reproduction in any medium, provided the original work is properly credited. The Creative Commons Public Domain Dedication waiver (http://creativecommons.org/publicdomain/zero/1.0/) applies to the data made available in this article, unless otherwise stated. 


\section{Background}

Despite the frequent occurrence of multiple parasite infections [1], little is known how concurrent parasite infections influence immune responsiveness in patients and few studies addressed the impact of helminth and intestinal protozoa co-infections on immune responses in children. The intensity and prevalence of helminth parasite infections is age-dependent with children being often the most affected [2,3], and poly-parasitism being more frequent than single parasite infections $[1,4,5]$. The existing epidemiological surveys on helminth co-infections in children indicate positive associations between schistosomes and soil transmitted helminths ( $\mathrm{STH}$ ) [5] which means high prevalence of mixed infections, as well as higher intensities of infection in co-infected patients [6]. For multiply infected patients, additive effects on the cellular reactivity and the down-modulation of cytokines are proposed [6]. In human mono-infections with STH an increased secretion of Th2-type cytokines associated with decreased cellular proliferation to specific parasite antigens and mitogens has been observed $[7,8]$. Protective Th2-type associated mechanisms have been suggested which may act distinctly during parasite migration leading to a reduction in numbers, size, and motility of migratory larvae [9-11] mediating a partial protection which will prevent an endless accumulation of adult helminthes parasites in the host [6]. In situations where several parasite species co-exist, where balanced and to some extent compromised immune responses are required, regulatory $\mathrm{T}$ cells and regulatory cytokines like IL-10 and IL-27 could represent the key components to prevent overwhelming inflammation and tissue destruction, but multiple and chronic infections may also drive immune responsiveness towards exhaustion. In poly-parasitized adults, parasitespecific cellular reactivity was significantly reduced in doubly infected individuals than in patients with a single filarial infection, and anti-parasite treatment greatly changed their cytokine and chemokine responses $[9,12,13]$. Generally accepted, Th2 type cytokines and chemokines play an essential role in the pathogenesis of allergic inflammation. Th2-type cytokines like IL-4, IL-5, IL-9 and IL-13 contribute to the pathophysiological conditions of allergy and asthma, and chemokines like eotaxin/CCL11, RANTES/CCL5 and monocyte chemoattractant proteins (MCPs) contribute decisively to the recruitment of basophil and eosinophil granulocytes as well as mast cells in tissues of allergic inflammation [14]. Chemokines attract Th2-type cells into bronchi and gut mucosal tissues in response to allergen exposure or intestinal helminthes parasite infection $[15,16]$. Intravascular parasite infections were described to ameliorate and even prevented allergen induced skin sensitization in humans [17] as well as airway hyper-reactivity in experimental animal studies [18]. Such an immune modulating capacities of pathogens has been extended to situations without active infection where exposure to environmental non-viable microbial products sufficed to reduce the occurrence of hay fever, atopic asthma, and atopic sensitization to environmental allergens [19] In children, poly-parasite infections with $S$. haematobium/S. mansoni, E. histolytica/E. dispar, and $N$. americanus generated prominent pro-inflammatory cytokine and chemokine responses, and anti-helminth treatment lessened inflammatory chemokine responses whilst the Th2 responsiveness in co-infected children increased [5]. Furthermore, long-term periodic anti-helminth treatments were associated with an increased prevalence of allergen skin test reactivity [20] and eczema symptoms [21].

In the present work, we analyzed in children infected with helminthes and protozoan parasites the cellular responses to protozoa or helminthes extracts and allergens, and observed distinct cytokine and chemokine production profiles; furthermore, in co-infected children prominent inflammatory chemokine and cytokine responses were observed together with enhanced allergen skin test reactivity.

\section{Methods}

\section{Study participants}

This study was conducted in the central region of Togo, Africa. All children examined were attending primary public schools in suburban quarters of the town of Sokodé. For stool and urine examinations, $50-\mathrm{mL}$ polypropylene tubes were distributed to the pupils and collected the next morning; diagnostic procedures were performed by the laboratory staff at the Centre Hospitalier Régional (CHR), Sokodé.

On the basis of the diagnostic results 4 infection groups were formed. All children who did not have a parasite infection and were apparently healthy (no fever, headache, vomiting, abdominal pain, diarrhea, dizziness, or skin lesions) were considered to be control children (Group G0 = NEG). Children with single infection Group G1, those with double infection Group G2 and with three and parasite infections are Group G3+.

In Tables 1 and 2 the children infection groups G0, G1, G2 and G3+ are listed from whom peripheral blood mononuclear cells $($ PBMC) were isolated $(n=87)$ and studied in vitro for cytokine and chemokine responses to parasite antigens and fungus and mite allergens. Demographic data and infection details from participating children are shown in Tables 1 and 2; the infection groups G0, G1, G2 and G3+ are listed by parasite species and abbreviations are indicated: $\mathrm{Na}=$ Necator americanus; $\mathrm{Eh}=$ Entamoeba histolytica/dispar; $\mathrm{Gl}=$ Giardia lamblia; $\mathrm{Hn}=$ Hymenolepis nana; $\mathrm{Sh}=$ Schistosoma haematobium; $\mathrm{Sm}=$ Schistosoma mansoni; Ti-Trichomonas intestinalis. Children from whom PBMC were isolated 
Table 1 Demographic data of children from whom peripheral blood mononuclear cells (PBMC) were isolated and PBMC used in cell culture experiments for the evaluation of cytokine and chemokine responses to parasite antigens and fungus and mite allergens

\begin{tabular}{llllll}
\hline & & $\mathbf{n}$ & Median age & Min age & Max age \\
\hline G0 = NEG (14) & $\mathrm{f}$ & 8 & 11 & 10 & 12 \\
& $\mathrm{~m}$ & 6 & 11 & 10 & 12 \\
G1 (32) & $\mathrm{f}$ & 15 & 10 & 9 & 13 \\
& $\mathrm{~m}$ & 17 & 12 & 10 & 13 \\
G2 (18) & $\mathrm{f}$ & 5 & 11 & 9 & 12 \\
& $\mathrm{~m}$ & 13 & 11 & 10 & 13 \\
G3+ (23) & $\mathrm{f}$ & 4 & 12 & 11 & 12 \\
& $\mathrm{~m}$ & 19 & 11 & 10 & 13 \\
\hline
\end{tabular}

Children were grouped according to the presence or absence of parasite infections; without parasite infection (Group $\mathrm{GO}=\mathrm{NEG}$ ), children with single infection (Group G1), with double (Group G2) and with poly-parasite infections (Group G3+). From children grouped by number of parasite infection (Abbreviations: $f / m=$ female/male).

were invited with their parents or legal guardians to the CHR Sokodé for blood sample collection. Children who showed signs of malaria (thick blood smears positive for Plasmodium species and fever), or who had diarrhea were excluded from the study. None of the children presented with $E$. histolytica trophozoites containing ingested red blood cells in stool samples, bloody stool, or clinical signs of invasive amoebiasis. Eight weeks after treatment, stool, urine, and blood samples from G0, G1, and G3+ children $(n=22)$ were re-examined in the same fashion as before treatment.

In Tables 3 and 4 the children infection groups G0, G1, G2 and G3+ are listed in whom skin prick test reactivity was evaluated $(n=509)$; demographic data and infection details from participating children are shown.

All children examined received a single dose of albendazole (400 mg), and those infected with S. haematobium or $S$. mansoni were treated with praziquantel according to the guidelines of the Togolese Ministry of Health. Diagnostic procedures to distinguish $E$. histolytica from $E$. dispar are not available at the laboratory facilities at the CHR, Sokodé, and treatment of E. histolytica/E. dispar is recommended on evidence of invasive amoebiasis (i.e., E. histolytica trophozoites containing ingested red blood cells in stool samples, bloody stool, or clinical signs of invasive amoebiasis).

Authorization and approval for the present work was granted by the Togolese Ministry of Health (292/99/MS/ $\mathrm{CAB}$ and $0407 / 2007 / \mathrm{MMS} / \mathrm{CAB} / \mathrm{DGS})$, the regional ministry of education (MENR/SG/DRERC/13.06.2001), the regional health authority (MS/DGS/DRS/RC/No.220 and MS/DGS/DRS/RC/No.261) and the Ethik Kommission at University Clinics Tübingen/Germany (No. 188/2008/
Table 2 Infections listed by parasite species in children from whom peripheral blood mononuclear cells (PBMC) were isolated and PBMC studied in vitro for cytokine and chemokine responses to parasite antigens and fungus and mite allergens

\begin{tabular}{|c|c|c|c|c|c|c|c|c|c|}
\hline G0 & NEG & & & & & & & 14 & $\mathrm{NEG}=16 \%$ \\
\hline G1 & & & & & & & Ss & 1 & \\
\hline G1 & & & & & & $\mathrm{Ti}$ & & 1 & \\
\hline G1 & & & & $\mathrm{Sm}$ & & & & 1 & \\
\hline G1 & & & Sh & & & & & 4 & \\
\hline G1 & & $\mathrm{Na}$ & & & & & & 4 & \\
\hline G1 & Eh & & & & & & & 21 & $\mathrm{G} 1=37 \%$ \\
\hline G2 & & & & & Gl & & Hn & 1 & \\
\hline G2 & & & Sh & $\mathrm{Sm}$ & & & & 1 & \\
\hline G2 & & $\mathrm{Na}$ & & & Gl & & & 2 & \\
\hline G2 & & $\mathrm{Na}$ & Sh & & & & & 2 & \\
\hline G2 & Eh & & & & & $\mathrm{Ti}$ & & 1 & \\
\hline G2 & Eh & & & & Gl & & & 4 & \\
\hline G2 & Eh & & & $\mathrm{Sm}$ & & & & 2 & \\
\hline G2 & Eh & & Sh & & & & & 1 & \\
\hline G2 & Eh & $\mathrm{Na}$ & & & & & & 4 & $G 2=21 \%$ \\
\hline G3+ & & $\mathrm{Na}$ & Sh & & Gl & & & 1 & \\
\hline G3+ & & $\mathrm{Na}$ & Sh & Sm & & & & 1 & \\
\hline G3+ & Eh & & & & $\mathrm{Gl}$ & $\mathrm{Ti}$ & & 1 & \\
\hline G3+ & Eh & & Sh & $\mathrm{Sm}$ & & & & 7 & \\
\hline G3+ & Eh & $\mathrm{Na}$ & & & & $\mathrm{Ti}$ & & 1 & \\
\hline G3+ & Eh & $\mathrm{Na}$ & & & & $\mathrm{Ti} \mathrm{Hr}$ & Un & 1 & \\
\hline G3+ & Eh & $\mathrm{Na}$ & & & Gl & $\mathrm{Ti}$ & & 1 & \\
\hline G3+ & Eh & $\mathrm{Na}$ & & $\mathrm{Sm}$ & & & & 3 & \\
\hline G3+ & Eh & $\mathrm{Na}$ & Sh & & & & & 4 & \\
\hline G3+ & Eh & $\mathrm{Na}$ & Sh & Sm & & & & 2 & \\
\hline \multirow[t]{2}{*}{ G3+ } & Eh & $\mathrm{Na}$ & Sh & $\mathrm{Sm}$ & & $\mathrm{Ti}$ & & 1 & $G 3=26 \%$ \\
\hline & & & & & & & & 87 & \\
\hline
\end{tabular}

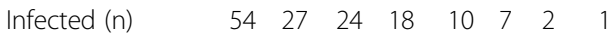

Infection (\%) $\quad \begin{array}{llllllll}62 & 31 & 28 & 21 & 11 & 8 & 2 & 1\end{array}$

Children without infection are Group G0 = NEG, children with single infection Group G1, those with double infection Group G2 and with three and parasite infections are Group G3+. Infections are listed by parasite species and abbreviations are shown: $\mathrm{Na}=$ Necator americanus; $\mathrm{Eh}=$ Entamoeba histolytica/dispar; $\mathrm{Gl}$ = Giardia lamblia; $\mathrm{Hn}=$ Hymenolepis nana; $\mathrm{Sh}=$ Schistosoma haematobium; Sm = Schistosoma mansoni; Ti-Trichomonas intestinalis).

BO2). Oral informed consent was given by all participating children, and written consent was provided by all parents or legal guardians after thorough explanation of the procedures, aims, and risks of the study; moreover, to ensure informed understanding, explanations were always given in the local language by the medical staff at the CHR, Sokodé. 
Table 3 Demographic data (age, sex) of children evaluated for skin prick test reactivity

\begin{tabular}{llllll}
\hline & & $\mathbf{n}$ & Median age & Min & Max \\
\hline G0 = NEG (287) & $\mathrm{f}$ & 121 & 11 & 7 & 12 \\
& $\mathrm{~m}$ & 166 & 11 & 9 & 12 \\
G1 (137) & $\mathrm{f}$ & 51 & 11 & 8 & 12 \\
& $\mathrm{~m}$ & 86 & 11 & 8 & 12 \\
G2 (58) & $\mathrm{f}$ & 25 & 12 & 9 & 12 \\
& $\mathrm{~m}$ & 33 & 12 & 9 & 12 \\
G3+ (27) & $\mathrm{f}$ & 9 & 12 & 11 & 12 \\
& $\mathrm{~m}$ & 18 & 11 & 10 & 12 \\
\hline
\end{tabular}

Children were grouped according to the presence or absence of parasite infections: without parasite infection (Group $\mathrm{GO}=\mathrm{NEG}$ ), with single infection (Group G1), double (Group G2) and with poly-parasite infections (Group G3+).

\section{Parasitological analysis}

For determination of intestinal helminth and protozoan infections, fresh stool samples $(0.5 \mathrm{~g})$ were mixed with saline and dispersed on 2 microscope slides covered with a $24 \times 48-\mathrm{mm}$ slide; samples were examined by laboratory technicians. All stool samples were examined using the Kato-Katz technique for quantification of helminth eggs per gram of stool (helm-TEST; Labmaster). To detect $S$. haematobium infection, $10 \mathrm{ml}$ of urine from each participant was filtered (polycarbonate membrane; pore size, $12 \mu \mathrm{m}$; Whatman); the filters were then examined under a microscope, and S. haematobium eggs were quantified. Thick blood smears were analyzed for the presence of Plasmodium species before and 8 weeks after anti parasite treatment of G0, G1, and G3+ children.

\section{Skin prick test examination}

Aspergillus fumigates and Dermatophagoides pteronysinus prick test solutions (Bencard Allergie $\mathrm{GmbH}$, München, Germany) were used. Allergens and positive histamine and negative saline controls were pricked onto the volar surface of the forearm, and reactions were recorded after 15 min. A skin prick test (SPT) weal cut-off diameters of $3 \mathrm{~mm}$ or larger was considered as a positive reaction. All tests were conducted by the same observer (RGG); reactions below this level were considered as non-specific and not reproducible.

\section{Isolation of peripheral blood mononuclear cells (PBMCs) and cell culture experiments}

Venous blood $(9 \mathrm{ml})$ was collected from participating children before and 8 weeks after anti-parasite treatments. PBMCs were isolated by ficoll-hypaque density gradient centrifugation at $340 \mathrm{~g}$ for $35 \mathrm{~min}$ at room temperature. Plasma samples were collected and frozen at $-20^{\circ} \mathrm{C}$ until further use. PBMCs were cultured in RPMI 1640 medium (Gibco) supplemented with $0.025 \mathrm{~mol} / \mathrm{l}$ HEPES buffer, $100 \mathrm{U} / \mathrm{ml}$ penicillin, $100 \mu \mathrm{g} / \mathrm{ml}$ streptomycin, and $0.25 \mu \mathrm{g} /$ $\mathrm{ml}$ amphotericin B (Sigma). The cell suspension was adjusted to $2,5 \times 10^{6}$ cells $/ \mathrm{ml}$ and $1,25 \times 10^{6} \mathrm{PBMC}$ were cultured in $0.5 \mathrm{ml}$ RPMI 1640 supplemented as described above plus $10 \%$ heat-inactivated fetal calf serum (Biochrom). PBMCs were cultured in 48-well plates at $37^{\circ} \mathrm{C}$ in $5 \% \mathrm{CO} 2$ with saturated humidity in the presence of either Entamoeba histolytica strain HM1 antigen (EhAg; $10 \mu \mathrm{g} / \mathrm{ml}$ ), Plasmodium falciparum schizont lysate (PfAg; $1 \times 10^{8}$ schizonts $/ \mathrm{ml}$ ), Schistosoma mansoni adult worm extract (SmAg; $10 \mu \mathrm{g} / \mathrm{ml}$ ), Echinococcus multilocularis metacestode extract (EmAg; $30 \mu \mathrm{g} / \mathrm{ml}$ ), Ascaris lumbricoides adult worm extract (AscAg; $5 \mu \mathrm{g} / \mathrm{ml}$ ), Lipopolysaccharid from Escherichia coli 026:B6 (LPS; $10 \mu \mathrm{g} / \mathrm{ml}$ ), Dermatophagoides pteronyssinus (Dp, $20 \mu \mathrm{g} / \mathrm{ml}$ ), Dermatophagoides farinae (Df, $20 \mu \mathrm{g} / \mathrm{ml}$ ), Aspergillus fumigatus (Af; $20 \mu \mathrm{g} / \mathrm{ml})$, Candida albicans (Ca; $20 \mu \mathrm{g} / \mathrm{ml}$ ) (Dp, Df, Af, and Ca extracts were all from Allergopharma, Reinbek, Germany), or PBMC were left unstimulated (baseline). Cell cultures were terminated after 48 hours, and cell-free supernatants were collected and stored below $-20^{\circ} \mathrm{C}$ until further use.

\section{Determination of cytokine and chemokine production in} cell culture supernatants

Quantitative enzyme-linked immune sorbent assay (ELISA) was performed with commercially available assays to determine in cell culture supernatants the levels of the cytokines IL-10, IL-27 and IL-33, as well as of the chemokines MIP3$\alpha / C C L 20$ and MIG/CXCL19 (Duo-Set; R\&D Minneapolis, MN, USA). Sample concentrations of each cytokine and chemokine were quantified from standard curves generated with recombinant chemokines/cytokines, and the lower limit for their detection was $30 \mathrm{pg} / \mathrm{ml}$ for IL-10, $170 \mathrm{pg} / \mathrm{ml}$ for IL-27, $25 \mathrm{pg} / \mathrm{ml}$ for IL-33, $15 \mathrm{pg} / \mathrm{ml}$ for MIP3- $\alpha / C C L 20$ and $60 \mathrm{pg} / \mathrm{ml}$ for MIG/CXCL9. ELISAs were performed as recommended by the manufacturer.

\section{Statistical analysis}

JMP software (versions 5.1; SAS Institute) was used for statistical analysis of data. Because of multiple comparisons, the level of significance was adjusted according to Bonferroni-Holm (alpha $=0.0018$ ). For the cytokine and chemokine analyses, differences between groups were determined after logarithmic transformation to stabilize the variance of data $(\log [\mathrm{pg} / \mathrm{ml}+1])$. Paired data from patients were evaluated by $t$-test and unpaired data of patient groups were compared using Wilcoxon's rank sum test.

\section{Results}

\section{Parasite infections in children}

For in vitro analyses of cellular responses in children, peripheral blood, stool and urine samples were collected 
Table 4 Parasite infections in children evaluated for skin prick test reactivity

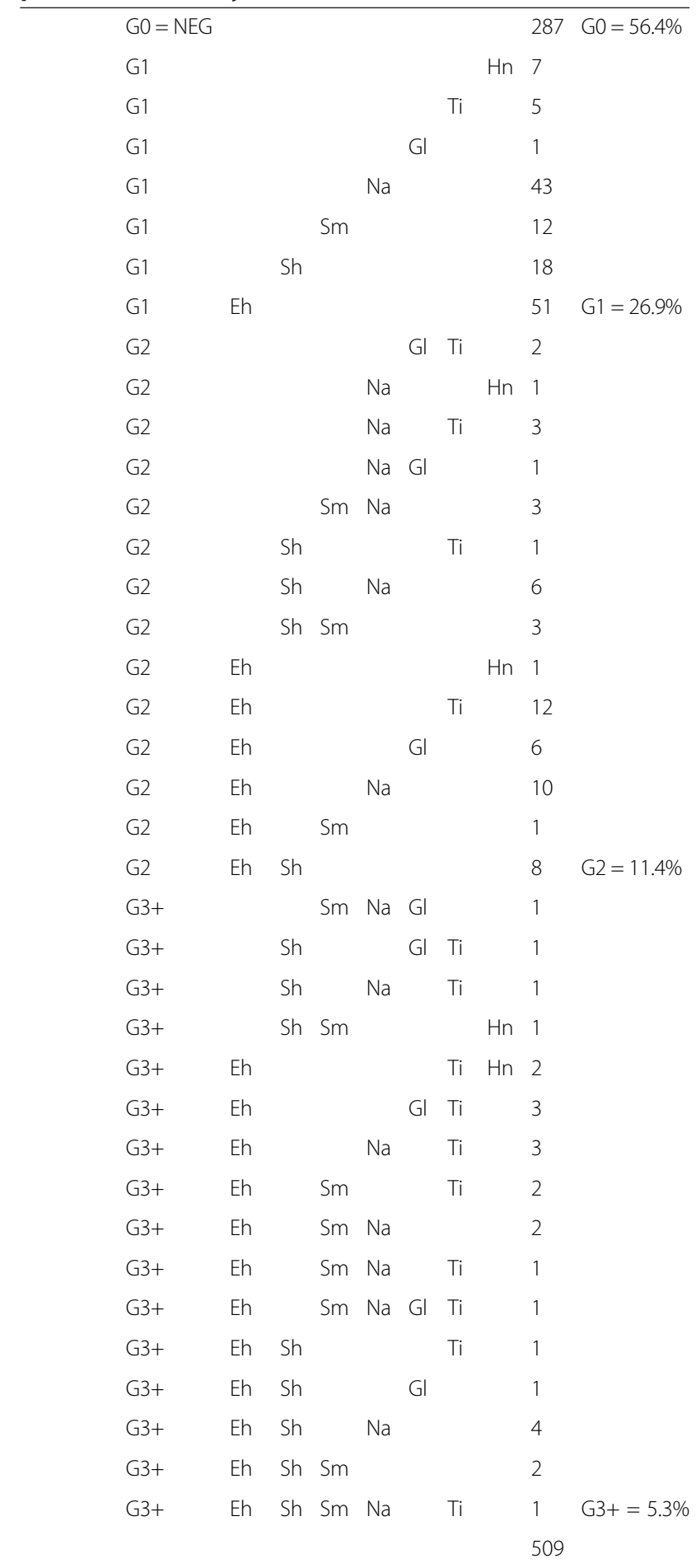

Infeted(n)

$\begin{array}{lllllll}112 & 48 & 30 & 81 & 17 & 39 & 12\end{array}$

Infection (\%)

Children were grouped according to presence or absence of infection and by parasite species. Without infection were Group $G 0=N E G$, children with single infection were Group G1, with double infection were Group G2 and with three and more parasite infections were Group G3+. Infections are listed by parasite species and abbreviations are shown: $\mathrm{Na}=$ Necator americanus; $\mathrm{Eh}=$ Entamoeba histolytica/dispar; $\mathrm{Gl}=$ Giardia lamblia; $\mathrm{Hn}=$ Hymenolepis nana; $\mathrm{Sh}=$ Schistosoma haematobium; Sm = Schistosoma mansoni; Ti-Trichomonas intestinalis. and examined from 87 children (Tables 1 and 2). With $62 \%(\mathrm{n}=54)$ Entamoeba histolytica/dispar was the most frequent parasite infection diagnosed, followed by hookworm $(31 \%, \mathrm{n}=27)$ while $28 \%(\mathrm{n}=24)$ and $21 \%(\mathrm{n}=18)$ were positive for Schistosoma haematobium or S. mansoni, and $16 \%(\mathrm{n}=14)$ were parasite infection-free. Giardia lamblia infection was present in $11 \%(\mathrm{n}=10)$, Hymenolepis nana eggs in 2\% $(\mathrm{n}=2)$ and Strongyloides stercoralis larvae were found in $\mathrm{n}=1(1 \%)$ of the participants. Singly infected were $37 \%(n=32), 21 \%$ of the children were positive for 2 $(\mathrm{n}=18)$ and $26 \%(\mathrm{n}=23)$ positive for 3 and more parasite species (Table 2).

Cellular cytokine and chemokine production of IL-10, IL-27, IL-33, MIP3- $\alpha / C C L 20$ and MIG/CXCL9 to parasite antigens and allergens

The cellular production of cytokines and chemokines in children was selectively inducible by parasite antigens and allergen extracts. Antigens of the intestinal protozoan Entamoeba histolytica (EhAg), cestode Echinococcus multilocularis (EmAg), mite-derived Dermatophagoides spp. (Dp, Df) and bacterial LPS were strong inducers of cellular IL-10 and MIP3- $\alpha /$ CCL20 release (Figure 1). In contrast, nematode Ascaris-, trematode Schistosoma- and protozoa Plasmodium-derived antigens and yeast Candida albicans (Ca) extracts selectively induced MIG/CXCL9, but did not activate IL-10 production by PBMC above the spontaneously released baseline levels (Figure 1). The cestode Echinococcus antigens activated IL-27 ( $<<0.04)$ whilst Ascaris extracts suppressed cellular IL-27 responses below $(\mathrm{p}<0.002)$ the spontaneous release (baseline), and in opposite, Ascaris extracts enhances enhanced IL-33 ( $\mathrm{p}=0.006)$ above baseline levels and Echinococcus antigens suppressed IL-33 $(\mathrm{p}=0.03)$ cellular release (data not shown).

\section{Cytokine and chemokine responses in singly and poly parasitized children}

In children with single and multiple parasite infections, the IL-10 responses to antigens and allergens were similarly expressed irrespective of the number and species of parasite infections, i.e. the levels of IL-10 as released by $\mathrm{PBMC}$ in $\mathrm{pg} / \mathrm{ml}$ from singly infected children did not further enhance with concurrent and additional parasite challenge (Figure 2). Following anti-parasite treatment, and without having achieved complete parasite elimination, the cellular production of IL-10 significantly diminished in the children (Figure 2). In the children infection groups, the amounts of IL-27 and IL-33 as produced by their PBMC increased in order $\mathrm{G} 0<\mathrm{G} 1<\mathrm{G} 3+$; the IL-27 production remained unchanged post anti-parasite treatment $(\mathrm{pT})$, whilst the cellular IL-33 production returned $\mathrm{pT}$ to the lowest levels as found in G0 children (Figure 3). Irrespective of the children infection groups 


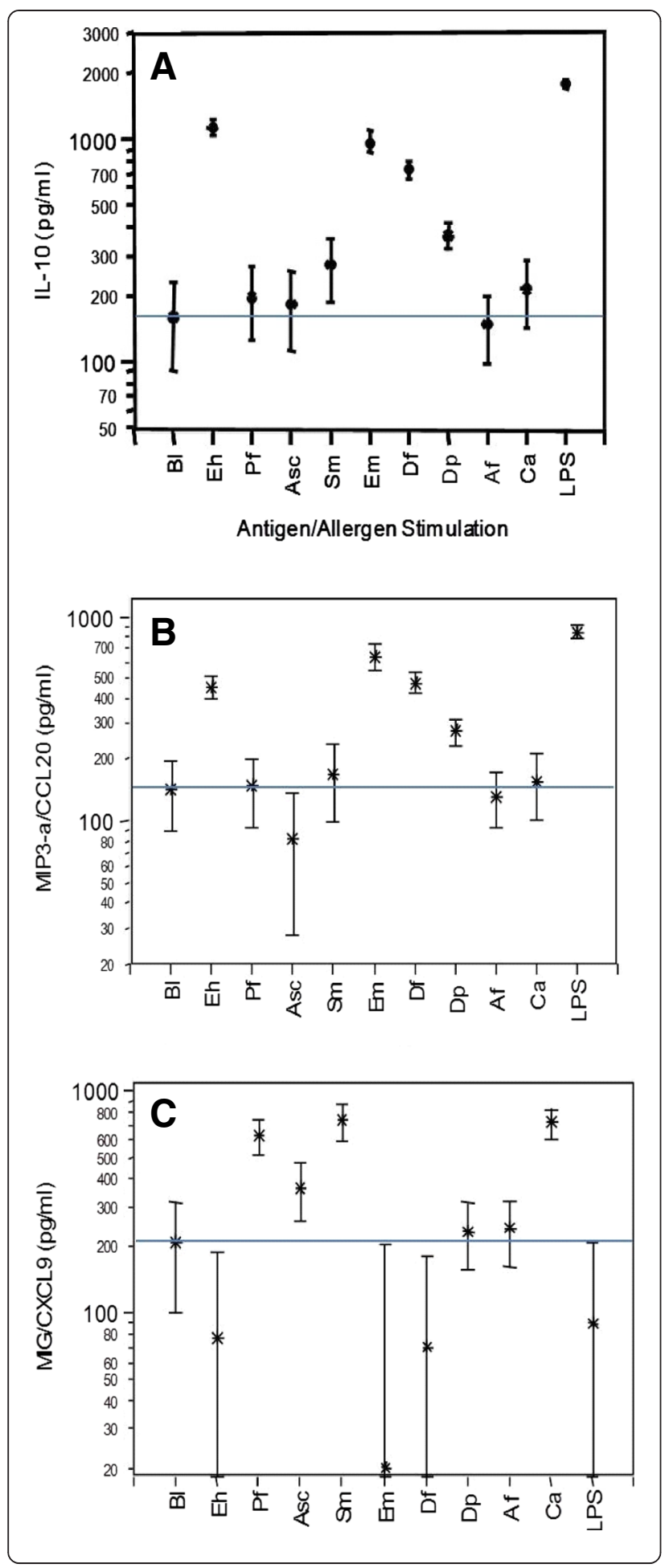

Figure 1 The inducible and spontaneous cellular production (in $\mathrm{pg} / \mathrm{ml}$ ) of interleukin 10 (IL-10) (Part A), Monocyte Inflammatory Protein 3-a (MIP-3a/CCL20) (Part B) and Monokine Inducible by Interferon- $\gamma$ (MIG/CXCL9) (Part C) by peripheral blood mononuclear cells (PBMC) from children is shown. Cellular cytokine or chemokine production was quantified by specific ELISA in cell culture supernatants after stimulation of PBMC for 48 hours with parasite-specific antigens from Entamoeba histolytica (Eh), Plasmodium falciparum (Pf), Ascaris lumbricoides (Asc), Schistosoma mansoni (Sm), Echinococcus multilocularis (Em), bacterial lipopolysaccharid (LPS) from Escherichia coli or allergen extracts from Dermatophagoides farinae (Df), Dermatophagoides pteronyssinus (Dp), Aspergillus fumigatus (Af) and Candida albicans (Ca). The spontaneous cytokine or chemokine releases by PBMC, i.e. cell cultures without antigen or allergen activation, are shown as baseline $(\mathrm{Bl})$, and the horizontal line indicates the mean spontaneous cytokine or chemokine release. Cellular production of IL-10, MIP-3a/CCL20 and MIG/CXCL9 are shown as means with the 95\% upper and lower confidence interval.

G0, G1, G3+, the release of MIP3- $\alpha$ /CCL20 remained at similar levels when PBMC were activated with $D$. pteronyssinus (Dp) and A. fumigatus (Af) (Figure 3). At 8 weeks post anti-parasite treatment (pT), the MIP3- $\alpha$ / CCL20 significantly diminished. For MIG/CXCL9, cellular release was low in G0 children, enhanced in G1 and G3+ children to some extent, and at 8 weeks post anti-parasite treatment (pT), it lessened to levels as found in the G0 children group (Figure 3).

\section{Cytokine and chemokine release with antigen and allergen co-stimulation}

In order to evaluate what effect simultaneous antigen and allergen exposure might exert on cellular cytokine and chemokine production, PBMC from G0, G1 and G3+ children were activated with allergen extracts and then exposed to helminth or protozoa antigens for 72 hours. While mite allergen extract strongly activated IL-10, IL-27 and MIP3- $\alpha / C C L 20$ production, IL-33 became suppressed (Figure 4). The co-culture of allergen-stimulated PBMC with helminth Ascaris antigens lessened the production of IL-27, IL-33 and MIP3- $\alpha$ /CCL20 to levels as observed with Ascaris antigens exposure alone; the IL-10 production as induced by $D$. pteronyssinus $(\mathrm{Dp})$ alone did not diminish in the presence of helminth antigen.

Exposure of PBMC to Entamoeba histolytica extract activated IL-10 above those levels as induced by $D$. pteronyssinus (Dp) alone (Figure 1), and the simultaneous exposure to both extracts further enhanced cellular IL-10 production. Protozoan E. histolytica antigen and the co-activation with mite allergens did not affect IL-27 and MIP3- $\alpha / C C L 20$ production by PBMC (not shown). Noteworthy, co-exposure of cells to Ascaris antigen in the presence of $D$. pteronyssinus $(\mathrm{Dp})$ allergen extracts suppressed the release of IL-33 by PBMC (Figure 4). 

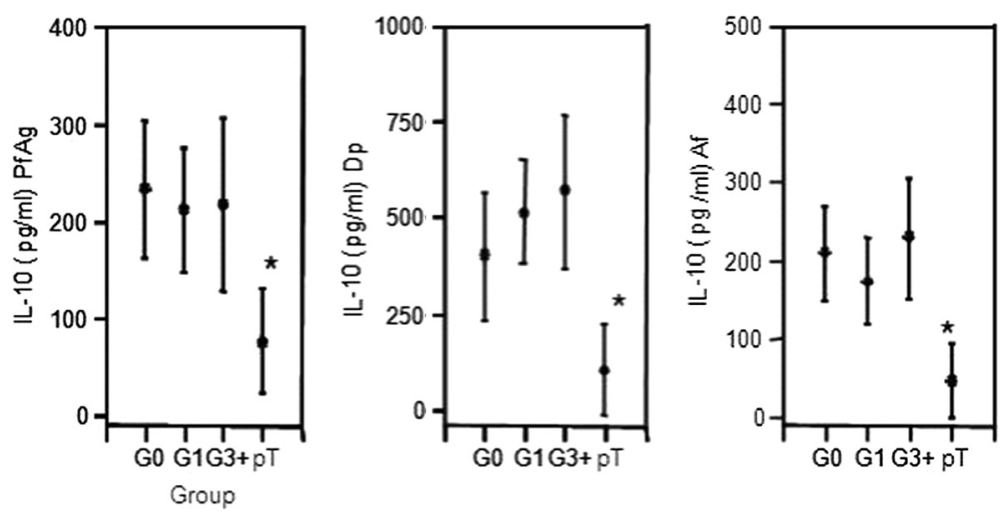

Figure 2 The cellular production of interleukin 10 (IL-10) by peripheral blood mononuclear cells (PBMC) is shown in children without parasite infection (Group G0, $n=11$ ), children with single infection (Group $\mathrm{G1}, \mathrm{n}=14$ ) and children with poly-parasite infections (Group $\mathbf{G} 3+, \mathbf{n}=7)$, and also, IL-10 production in children at 8 weeks post anti-parasite treatment $(\mathbf{p T}, \mathbf{n}=19)$ is given. The release of IL-10 by PBMC was quantified by cytokine specific ELISA in cell culture supernatant of PBMC activated with antigens from P. falciparum (Pf), with D. pteronyssinus (Dp) or with A. fumigatus (Af) is shown in G0, G1, G3+ children before and 8 weeks post anti-parasite treatment (pT). Cellular production of IL-10 is shown as means with the $95 \%$ upper and lower confidence interval. The level of significance was adjusted according to Bonferroni-Holm, and significant differences between groups are indicated as *P $\leq 0.002$.

\section{Skin prick test responses to allergens in singly and parasite} co-infected children

For analysis of skin prick test (SPT) responsiveness in singly, doubly and poly-parasitized children, stool and urine samples were collected and examined from $\mathrm{n}=509$ pupils (Tables 3 and 4). Skin prick test responses to Aspergillus fumigatus (Af) and Dermatophagoides spp. (Df) were evaluated in $\mathrm{n}=287$ non-infected, in $\mathrm{n}=137$ singly, in $\mathrm{n}=58$ doubly and $n=27$ poly-parasitized children (Table 4). In non-infected children skin prick test responses developed less often $(\mathrm{p}<0.0001)$ than in those with parasite infections (Figure 5). In non-infected children $23 \%$, in singly infected $47 \%$, in doubly parasite-infected $53 \%$ and those with 3 and more parasite infections $56 \%$ developed positive $\mathrm{SPT}$ reactions to Af and Df extracts. Notable, the presence or absence of Entamoeba histolytica/dispar or hookworm infections did not interact with SPT responses while Schistosoma spp. infections were associated with an enhanced SPT reactivity to Af and Df extracts $(\mathrm{p}=0.007)$.

\section{Discussion}

The present study revealed in children infected with helminthes and protozoan parasites distinctive cellular cytokine and chemokine response profiles to antigens of protozoa, helminthes, bacteria and allergens. The intestinal protozoa Entamoeba histolytica, bacterial LPS and mite-allergens stimulated the production of the regulatory cytokine IL-10 and monocyte-inflammatory MIP3- $\alpha$ / CCL20. The Plasmodium, helminthes and Candida extracts strongly induced the cellular release of MIG/
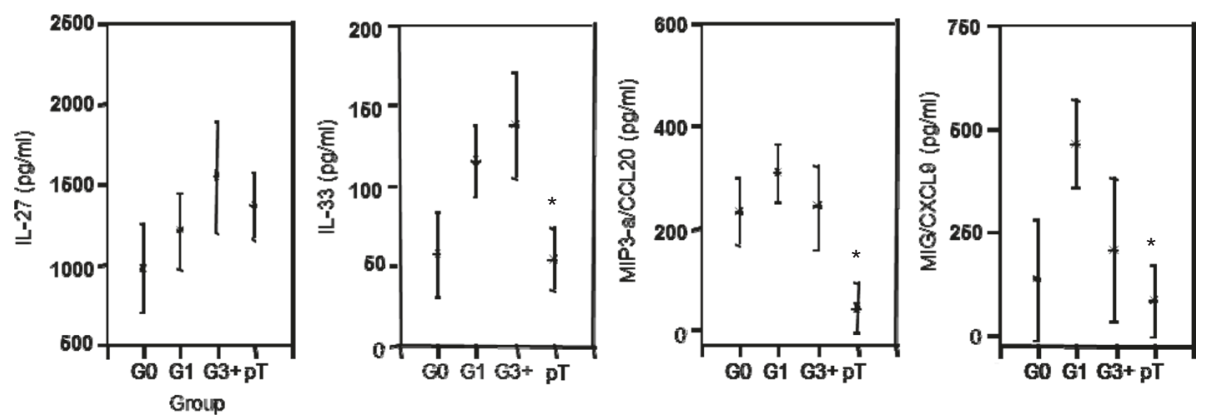

Figure 3 The cellular release of IL-27, IL-33, MIP3-a/CCL20 and MIG/CXCL9 in response to allergen extracts of Dermatophagoides pteronyssinus (Dp) and Aspergillus fumigatus (Af), in singly and poly-parasitized children; children without parasite infection (Group $\mathrm{G0}, \mathrm{n}=11$ ), children with single infection (Group $\mathrm{G1}, \mathrm{n}=14$ ) and children with poly-parasite infections (Group $G 3+, n=7$ ), and the cytokine and chemokine production in children at 8 weeks post anti-parasite treatment $(\mathrm{pT}, \mathbf{n = 1 9})$ is shown. The cytokine and chemokine responses to the extracts of $D$. pteronyssinus (Dp) and A. fumigatus (Af) are merged. The cellular productions of IL-10, IL-27, MIP-3a/CCL20 and MIG/CXCL9 are shown as means with the $95 \%$ upper and lower confidence interval. The level of significance was adjusted according to Bonferroni-Holm, and significant differences between groups are indicated as * $\mathrm{P} \leq 0.002$. 

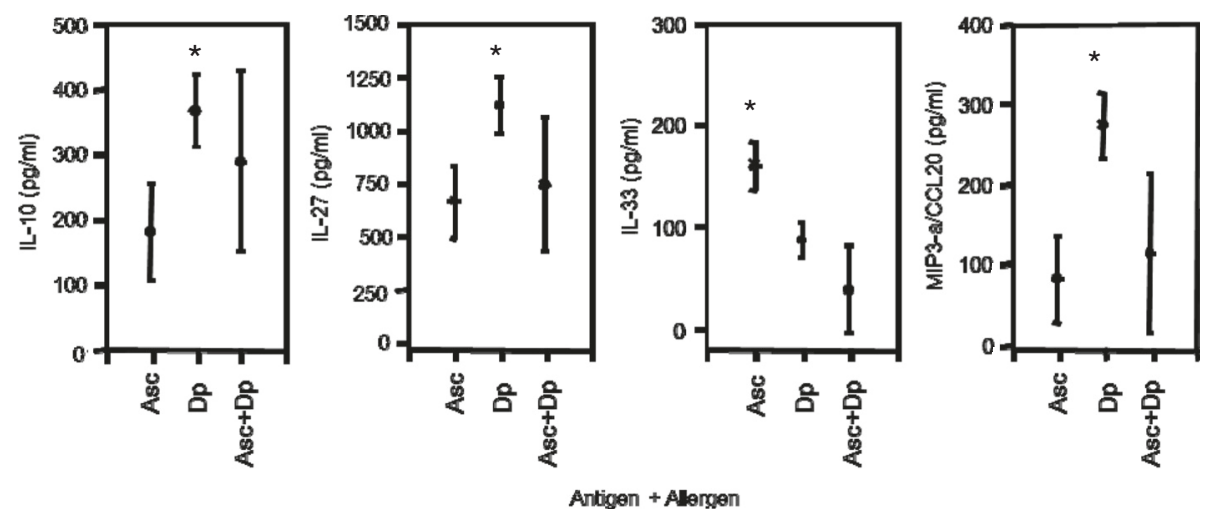

Figure 4 Cellular release (in pg/ml) of IL-10, IL-27, IL-33 and MIP3-a/CCL20 by PBMC activated alone with helminth-derived Ascaris lumbricoides (Asc) or allergen extracts from Dermatophagoides pteronyssinus (Dp) or after co-activation with Asc antigen and DP allergen. PBMC were isolated from singly and poly-parasitized children and cultured in vitro for 48 hours. Cellular production of IL-10, IL-27, IL-33, MIP-3a/CCL20 and MIG/CXCL9 are shown as means with the $95 \%$ upper and lower confidence interval. The level of significance was adjusted according to Bonferroni-Holm, and significant differences between groups are indicated as * $\leq 0.002$.

CXCL9, a monokine inducible by IFN- $\gamma$, while proinflammatory IL-33 was inducible by Ascaris and Echinococcus antigens only.

The IL-10 production as observed in infection-free children (group G0) did not further enhance with double (G2) or poly-parasite (G3+) infections, however, it diminished post anti-parasite treatment. This we have similarly observed in hookworm, filaria and E. histolytica co-infected adults [12]. The regulatory cytokine IL-10 will modulate Th1-type responses to antigens by depressing pro-inflammatory TNF, IFN- $\gamma$ and IL-12p70 [22] contributing to active and general immune suppression and

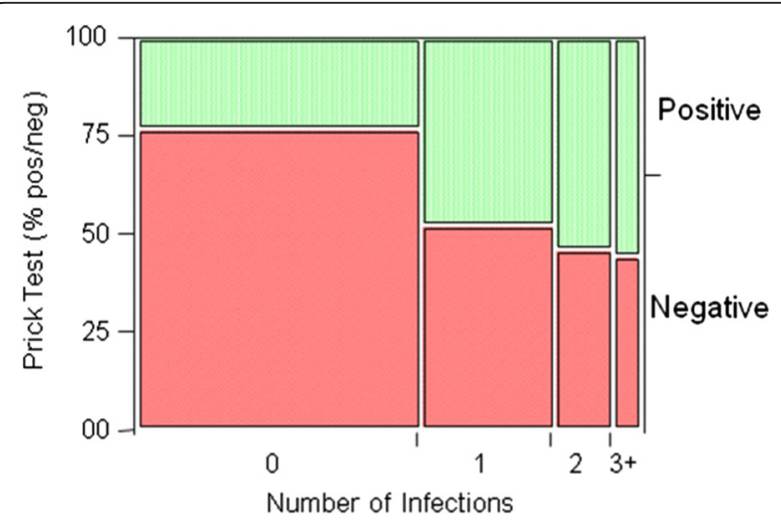

Figure 5 Skin prick test reactivity (positive/negative) in children (total $n=509$ ) to Aspergillus fumigates and Dermatophagoides pteronysinus prick test solutions were evaluated in $n=287$ non-infected (G0), in $n=137$ singly (G1), in $n=58$ doubly (G2) and $\mathbf{n}=\mathbf{2 7}$ poly-parasitized (G3+) children. Allergens and positive histamine and negative saline controls were pricked onto the volar surface of the forearm, and reactions were recorded after $15 \mathrm{~min}$. A skin prick test (SPT) weal cut-off diameters of $3 \mathrm{~mm}$ or larger was considered as a positive reaction. ( $p<0.0001$ between $\mathrm{G} 0$ and $\mathrm{G} 1$ ). preventing inflammatory disease manifestations [23-25], but elevated IL-10 production levels may also facilitate the persistence of pathogens. Concurrent infections with several parasite species may counteract the regulatory effects of IL-10, notably when pathogens enhance the production of inflammatory cytokines and chemokines [26], and therefore, not singular cytokines but rather distinct cytokine response profiles may define the expression of immunity and severity of disease [27].

As a cytokine with regulatory capacity, IL-27 will initiate IFN- $\gamma$ responses and promote IL-10 synthesis by regulatory T cells, then attenuate Th2 and Th17 cells [28] and depress pro-inflammatory cytokines and chemokines $[29,30]$. In children, the production levels of regulatory IL-27 and also of inflammatory IL-33 rose with the number of infections $(\mathrm{G} 0<\mathrm{G}<\mathrm{G} 3+)$ and these responses were inducible by mite-allergen extracts. IL-27 was shown to be a key regulator of IL-10 and IL-17 production by human CD4+ T cells thus providing a dual regulatory mechanism to control autoimmunity and tissue inflammation [28-31]. The cytokine IL-33 was suggested to function as an "alarmin" [32]; in infants with severe malaria IL-33 levels were found enhanced, IL-33 concentrations in plasma correlated positively with parasite densities, and IL-33 diminished strongly following $P$. falciparum clearance [33].

The cellular production of the pro-inflammatory chemokines MIP3- $\alpha / C C L 20$ and MIG/CXCL9 was inducible by protozoan parasite antigens and mite and yeast allergens, and this wide range of responsiveness to allergens and parasite antigens may augment inflammatory cellular responsiveness. Indeed, the macrophage inflammatory protein MIP3- $\alpha / C C L 20$ and the monokine induced by interferon-gamma MIG/CXCL9 were found elevated in infants with severe malaria [33] and in patients with 
atopic dermatitis [34,35]. In contrast, helminthes parasites are considered as masterful regulators of specific immune responses [11,36,37], helminth parasite-induced IL-10 may modulate atopic responses in schistosoma-infected children [17], and in filaria-infected mice regulatory $\mathrm{T}$ cells will inhibit atopic and airway hyper-reactivity following allergen exposure [18].

Here, in children exposed to and infected with several parasite species not only regulatory IL-10 and antiinflammatory IL-27 was produced by their PBMC, but also pro-inflammatory IL-33, MIP-3a/CCL20 and MIG/ CXCL9 responses were observed, and with an increasing number of parasite infections these pro-inflammatory cytokines and chemokines responses enhanced.

Helminthes infections will active prominent Th2-type cytokines and immune regulatory processes [23] and chronic geo-helminthes infection with high total $\operatorname{IgE}$ and anti-Ascaris IgG4 may reduce the risk of atopy in schoolage children [38], but parasite infections do not in general protect against asthma while hookworm infection may reduce the risk of this disease [39]. In the present study none of the participating children was infected with Ascaris lumbricoides and it should be considered that hookworm and S. haematobium and S. mansoni infections will cause intestinal and urinary tract tissue damage resulting in bloody urines and mostly occult blood in stools; these helminthes will always cause gastrointestinal inflammation. As previously observed, in infants and children with helminth and protozoa infections regulatory cytokine and chemokine responses were not evolved to levels as observed in adults [40], and such immune response profiles may develop with ageing and repeated episodes of exposure and persistent parasite infections. In the present study, allergen-specific positive skin prick test (SPT) responses were more frequent in co-infected children than in infection-free pupils suggesting that parasite co-infections may have triggered or even amplified pro-inflammatory reactivity to allergens.

Furthermore, in the present study we could show that helminthes and protozoan parasite antigen extracts will influence cellular in vitro responses to allergens. When PBMC were simultaneously stimulated with Ascaris lumbricoides antigens and allergen extracts the production of IL-27, IL-33 and MIP3- $\alpha / C C L 20$ lessened to levels as observed with Ascaris antigen exposure alone, suggesting anti-inflammatory effects mediated by the Ascaris antigen extract. The potential of Ascaris antigen to dampen the cellular production of pro-inflammatory cytokines and chemokines, while regulatory IL-10 remained unaffected, may help to reduce inflammation and hyper reactivity responses. In contrast, the protozoan Entamoeba antigen extract enhanced IL-10, lessened the IL-33 release and left IL-27 and MIP3- $\alpha / C C L 20$ production unaffected, again disclosing divergent immune activation patterns to parasite antigen extracts. Thus, the molecular composition of individual parasite species, here helminthes or protozoa, will stimulate distinct cytokine and chemokine responses which may also influence immune responsiveness to allergens.

\section{Conclusions}

In children co-infected with hookworm, schistosomes and intestinal protozoan parasites their PBMC will generate mixed cytokine and chemokine response profiles to parasite antigens and allergens. The molecular composition of individual parasite or allergen extracts will distinctively stimulate regulatory as well as pro-inflammatory chemokines and cytokines, and with an increasing number of parasite infections pro-inflammatory cytokines and chemokines responses enhanced, while regulatory cytokine production remained stable. In poly-parasitized children skin prick test reactivity to allergens extracts was highest suggesting that parasite co-infections may have triggered or amplified their atopic responsiveness.

\section{Competing interest}

The authors declare that they have no competing interest.

\section{Authors' contributions}

$J H, C J L, X H, R G G$, PTS and CK conceived, designed and performed the experiments. RGG, JH, AA, YFA, and PTS recruited, examined and treated patients. JH, PTS, CJL, XH and CK analyzed the data. JH, PTS, CJL, XH and CK wrote the manuscript. All authors read and approved the final manuscript.

\section{Acknowledgements}

We kindly thank all parents and children who participated in the present work. For their expert assistance we thank the medical assistants and nurses at the Pediatric Ward at the Centre Hospitalier Regional (CHR) in Sokodé in Togo. The work was supported by the Bundesministerum für Bildung und Forschung (BMBF grant 01KA1008) and the Commission of the European Community FP7 Project (Grant Acronym E-PIAF \#242131).

We acknowledge support by Deutsche Forschungsgemeinschaft and Open Access Publishing Fund of Tübingen University.

\section{Author details}

${ }^{1}$ Institute for Tropical Medicine, University Clinics Tübingen, Wilhelmstraße 27, 72074 Tübingen, Germany. ${ }^{2}$ Institut National d'Hygiène - Onchocerciasis Reference Laboratory, Sokodé, Togo. ${ }^{3}$ Centre Hospitalier Régional, Service Pédiatrie, Sokodé, Togo.

Received: 27 June 2013 Accepted: 4 January 2015

Published online: 20 January 2015

\section{References}

1. Pennycook A, Openshaw P, Hussell T. Partners in crime: co-infections in the developing world. Clin Exp Immunol. 2000;122:296-9.

2. Bethony J, Brooker S, Albonico M, Geiger SM, Loukas A, Diemert D, et al. Soil-transmitted helminth infections: ascariasis, trichuriasis, and hookworm. Lancet. 2006;367:1521-32.

3. Pullan R, Brooker $\mathrm{S}$. The health impact of polyparasitism in humans: are we under-estimating the burden of parasitic diseases? Parasitology. 2008;135:783-94.

4. Buck AA, Anderson RI, MacRae AA. Epidemiology of poly-parasitism. II. Types of combinations, relative frequency and associations of multiple infections. Tropenmed Parasitol. 1978:29:137-44.

5. Hamm DM, Agossou A, Gantin RG, Kocherscheidt L, Banla M, Dietz K, et al. Coinfections with Schistosoma haematobium, Necator americanus, and Entamoeba histolytica/Entamoeba dispar in children: chemokine and cytokine responses and changes after antiparasite treatment. J Infect Dis. 2009;199:1583-91. 
6. Geiger SM. Immuno-epidemiology of Schistosoma mansoni infections in endemic populations co-infected with soil-transmitted helminths: present knowledge, challenges, and the need for further studies. Acta Trop. 2008;108:118-23.

7. Geiger SM, Massara CL, Bethony J, Soboslay PT, Carvalho OS, Corrêa-Oliveira R. Cellular responses and cytokine profiles in Ascaris lumbricoides and Trichuris trichiura infected patients. Parasite Immunol. 2002;24:499-509.

8. Geiger SM, Massara CL, Bethony J, Soboslay PT, Corrêa-Oliveira R. Cellular responses and cytokine production in post-treatment hookworm patients from an endemic area in Brazil. Clin Exp Immunol. 2004;136:334-40.

9. Quinnell RJ, Pritchard DI, Raiko A, Brown AP, Shaw MA. Immune responses in human necatoriasis: association between interleukin-5 responses and resistance to re-infection. J Infect Dis. 2004;190:430-8.

10. Jackson JA, Turner JD, Rentoul L, Faulkner H, Behnke JM, Hoyle M, et al. T helper cell type 2 responsiveness predicts future susceptibility to gastrointestinal nematodes in humans. J Infect Dis. 2004;190:1804-11.

11. Maizels RM, Balic A, Gomez-Escobar N, Nair M, Taylor MD, Allen J. Helminth parasites-masters of regulation. Immunol Rev. 2004;201:89-116.

12. Soboslay PT, Hamm DM, Pfafflin F, Fendt J, Banla M, Schulz-Key H. Cytokine and chemokine responses in patients co-infected with Entamoeba histolytical dispar, Necator americanus and Mansonella perstans and changes after anti-parasite treatment. Microbes Infect. 2006;8:238-47.

13. Mai CS, Hamm DM, Banla M, Agossou A, Schulz-Key H, Heuschkel C, et al. Onchocerca volvulus-specific antibody and cytokine responses in onchocerciasis patients after 16 years of repeated ivermectin therapy. Clin Exp Immunol. 2007;147:504-12.

14. Romagnani S. Cytokines and chemoattractants in allergic inflammation. Mol Immunol. 2002;38:881-5.

15. Panina-Bordignon P, D'Ambrosio D. Chemokines and their receptors in asthma and chronic obstructive pulmonary disease. Curr Opin Pulm Med. 2003:9:104-10.

16. Stein LH, Redding KM, Lee JJ, Nolan TJ, Schad GA, Lok JB, et al. Eosinophils utilize multiple chemokine receptors for chemotaxis to the parasitic nematode Strongyloides stercoralis. J Innate Immun. 2009;1:618-30.

17. van den Biggelaar $A H$, van Ree $R$, Rodrigues LC, Lell B, Deelder AM, Kremsner PG, et al. Decreased atopy in children infected with Schistosoma haematobium: a role for parasite-induced interleukin-10. Lancet. 2000;356:1723-7.

18. Dittrich AM, Erbacher A, Specht S, Diesner F, Krokowski M, Avagyan A, et al. Helminth infection with Litomosoides sigmodontis induces regulatory $T$ cells and inhibits allergic sensitization, airway inflammation, and hyperreactivity in a murine asthma model. J Immunol. 2008;180:1792-9.

19. Braun-Fahrländer C, Riedler J, Herz U, Eder W, Waser M, Grize L, et al. Environmental exposure to endotoxin and its relation to asthma in school-age children. N Engl J Med. 2002;347:869-77.

20. van den Biggelaar AH, Rodrigues LC, van Ree R, van der Zee JS, Hoeksma-Kruize YC, Souverijn JH, et al. Long-term treatment of intestinal helminths increases mite skin-test reactivity in Gabonese school children. J Infect Dis. 2004;189:892-900.

21. Endara P, Vaca M, Chico ME, Erazo S, Oviedo G, Quinzo I, et al. Long-term periodic anthelmintic treatments are associated with increased allergen skin reactivity. Clin Exp Allergy. 2010;40:1669-77.

22. Luty AJ, Perkins DJ, Lell B, Schmidt-Ott R, Lehman LG, Luckner D, et al. Low interleukin-12 activity in severe Plasmodium falciparum malaria. Infect Immun. 2000:68:3909-15.

23. Smits HH, Yazdanbakhsh M. Chronic helminth infections modulate allergen-specific immune responses: protection against development of allergic disorders? Ann Med. 2007;39:428-39.

24. Smits HH, Everts B, Hartgers FC, Yazdanbakhsh M. Chronic helminth infections protect against allergic diseases by active regulatory processes. Curr Allergy Asthma Rep. 2010;10:3-12.

25. Soboslay PT, Geiger SM, Weiss N, Banla M, Lüder CGK, Dreweck CM, et al. The diverse expression of immunity in humans at distinct states of Onchocerca volvulus infection. Immunology. 1997;90:592-9.

26. Oliveira SH, Lukacs NW. The role of chemokines and chemokine receptors in eosinophil activation during inflammatory allergic reactions. Braz J Med Biol Res. 2003;6:1455-63.

27. Sinha S, Qidwai T, Kanchan K, Jha GN, Anand P, Pati SS, et al. Distinct cytokine profiles define clinical immune response to falciparum malaria in regions of high or low disease transmission. Eur Cytokine Netw. 2010;21:232-40.
28. Murugaiyan G, Mittal A, Lopez-Diego R, Maier LM, Anderson DE, Weiner HL. $\mathrm{IL}-27$ is a key regulator of $\mathrm{IL}-10$ and IL-17 production by human CD4+ T cells. J Immunol. 2009;183:2435-43.

29. Sturmhofer SJ, Hunter CA. Advances in understanding the anti-inflammatory properties of IL-27. Immunol Lett. 2008;117:123-30.

30. Yoshida H, Nakaya M, Miyazaki Y. Interleukin 27: a double-edged sword for offense and defense. J Leukoc Biol. 2009;86:1295-303.

31. Hu S, Wong CK, Lam CW. Activation of eosinophils by IL-12 family cytokine IL-27: Implications of the pleiotropic roles of IL-27 in allergic responses. Immunobiology. 2011;216:54-65.

32. Moussion C, Ortega N, Girard JP. The IL-1-like cytokine IL-33 is constitutively expressed in the nucleus of endothelial cells and epithelial cells in vivo: a novel 'alarmin'? PLOS ONE. 2008;3:e3331.

33. Ayimba E, Hegewald J, Ségbéna AY, Gantin RG, Lechner CJ, Agossou A, et al. Proinflammatory and regulatory cytokines and chemokines in infants with uncomplicated and severe Plasmodium falciparum malaria. Clin Exp Immunol. 2011;166:218-26.

34. Nakayama T, Fujisawa R, Yamada H, Horikawa T, Kawasaki H, Hieshima K, et al. Inducible expression of a CC chemokine liver- and activation-regulated chemokine (LARC)/macrophage inflammatory protein (MIP)-3 alpha/CCL20 by epidermal keratinocytes and its role in atopic dermatitis. Int Immunol. 2001;13:95-103.

35. Shimada Y, Takehara K, Sato S. Both Th2 and Th1 chemokines (TARC/CCL17, MDC/CCL22, and MIG/CXCL9) are elevated in sera from patients with atopic dermatitis. J Dermatol Sci. 2004;34:201-8.

36. Maizels RM, Yazdanbakhsh M. Immune regulation by helminth parasites: cellular and molecular mechanisms. Nat Rev Immunol. 2003;3:733-44.

37. Harnett W, Harnett MM. Parasitic nematode modulation of allergic disease. Curr Allergy Asthma Rep. 2008;8:392-7.

38. Cooper PJ, Chico ME, Rodrigues LC, Ordonez M, Strachan D, Griffin GE, et al. Reduced risk of atopy among school-age children infected with geohelminth parasites in a rural area of the tropics. J Allergy Clin Immunol. 2003;111:995-1000.

39. Leonardi-Bee J, Pritchard D, Britton J. Asthma and current intestinal parasite infection: systematic review and meta-analysis. Am J Respir Crit Care Med. 2006;174:514-23.

40. Lechner CJ, Komander K, Hegewald J, Huang X, Gantin RG, Soboslay PT, et al. Cytokine and chemokine responses to helminth and protozoan parasites and to fungus and mite allergens in neonates, children, adults, and the elderly. Immun Ageing. 2013;10(1):29.

\section{Submit your next manuscript to BioMed Central and take full advantage of:}

- Convenient online submission

- Thorough peer review

- No space constraints or color figure charges

- Immediate publication on acceptance

- Inclusion in PubMed, CAS, Scopus and Google Scholar

- Research which is freely available for redistribution 